\title{
IGUALDAD Y DESIGUALDAD DE GÉNERO EN LOS IMAGINARIOS SOCIALES DEL AMOR DE PAREJA HETEROSEXUAL
}

\author{
Ana Dolores Verdú Delgado \\ anad.verdu@yahoo.es \\ Universidad Miguel Hernández de Elche
}

Recibido: 10-02-2014

Aceptado: 21-03-2014

\section{Resumen}

En las últimas décadas las relaciones de género han experimentado profundas transformaciones en España, paralelamente a la lucha por la igualdad, como valor institucional, y a los cambios producidos en los modos de organizar la vida íntima. No obstante, son muchos los estudios que detectan la importante influencia que todavía tiene el sexismo en los imaginarios del amor de la población más joven. Este artículo parte de la revisión de algunos de estos estudios para analizar posteriormente datos provenientes de una encuesta distribuida en centros universitarios de la provincia de Alicante. Los resultados de esta encuesta señalarían la mayor presencia de igualdad en este contexto con respecto a la adolescencia, detectando asimismo diferencias de género en determinados aspectos.

Palabras clave: Imaginarios sociales del amor, pareja, igualdad, desigualdad de género.

\begin{abstract}
In recent decades gender relations in Spain have undergone profound transformations in parallel with the fight for equality, as an institutional value, and the changes that have taken place with regard to the organisation of private life. However, many studies have detected that sexism continues to have a considerable influence among the younger population in their constructs of love. This article begins by reviewing some of these studies before analysing the data from a study distributed in universities of the province of Alicante. The results of this survey appear to indicate a greater presence of equality in this context when compared to teenagers, and gender differences are also detected in certain aspects.
\end{abstract}

Key words: Constructs of love, couple, equality, gender inequality. 


\section{Introducción: Aprendizaje de la desigualdad de género en la adolescencia}

Cuando los estudios de género prestan atención al modo en que la desigualdad se expresa en la experiencia del amor, un objetivo fundamental es comprender el papel que las creencias sobre éste ejercen en el contexto de las relaciones de pareja heterosexuales. La hipótesis de la que parten los trabajos que se preocupan por este tema es que, en la medida en que el imaginario colectivo del amor contenga idealizaciones de lo femenino y lo masculino dependientes de un sistema de género asimétrico, será frecuente que la pareja heterosexual se construya sobre la base de la desigualdad.

Las investigaciones que abarcan esta cuestión en España se han interesado principalmente por la población adolescente. La adolescencia representa una etapa peculiar en lo que se refiere al amor y al género, pues en ella tienen lugar frecuentemente las primeras relaciones de amor/sexo entre chicas y chicos al tiempo que adquiere vital importancia la manifestación de los roles sexuales como necesidad de responder a una nueva identidad. Especialmente en la cultura occidental, la adolescencia se vive como una edad de ruptura dramática con respecto a la etapa infantil, sobre todo en lo que respecta a la adopción de conductas de género típicas, fenómeno fomentado por una socialización que tiende a remarcar los aspectos vinculados a la sexualidad como parte esencial de la identidad.

Además, se ha de tener en cuenta que la población joven está sobreexpuesta, por el tipo de ocio mediatizado al que tiene acceso, a nuevas formas de sexismo que coexisten junto con el avance socio-político en igualdad y derechos. La asunción de papeles sexuales rígidos dentro de una ideología que normaliza la desigualdad y la violencia está muy presente en los productos culturales que forman parte del ocio de la juventud (videojuegos, Internet, televisión y revistas, principalmente). Este aspecto determina inevitablemente la forma de sus primeras experiencias en el amor y la sexualidad, algo que aparece reflejado en diferentes estudios con jóvenes.

Por ejemplo, algunas de estas investigaciones coinciden en que la expresión de la desigualdad en lo relacional-afectivo proviene de la reproducción no consciente del sexismo en los guiones amorosos de género. Charo Altable encuentra que estos guiones divergen en chicas y chicos, notando que los de ellas están más afectados por el romanticismo, el deseo de ser salvadas y el amor como proyecto principal en su identidad, mientras que los de ellos lo estarían en mayor medida por imágenes pornográficas (1991: 38). 
Otros elementos que aparecen con frecuencia en el imaginario del amor de la adolescencia en estos estudios son: la creencia en que las diferencias de género se justificarían por la biología, entendiendo así que los hombres serían más agresivos por naturaleza (Díaz Perera, 2009), y la asociación implícita del amor-pasión y la violencia, hasta el punto de que "un $80 \%$ de las chicas y un $75 \%$ de los chicos no relacionan la falta de amor con el maltrato. Piensan que se puede agredir, hacer sufrir y causar daño, a alguien a quien queremos" (Meras, 2003: 143).

Siguiendo también estas líneas de análisis, trabajos sobre aprendizaje emocional en la adolescencia detectan una pauta de género en las diferencias expresadas por parte de chicos y chicas en el contexto de sus primeras relaciones de pareja. Según Genoveva Sastre y Monserrat Moreno, las chicas empezarían a experimentar en la adolescencia, en sus relaciones con los chicos, altos niveles de sufrimiento (expresado en decepción, abandono, infidelidad e incluso maltrato) mientras que los chicos vivirían sus primeras relaciones con relativa tranquilidad, sin que la decepción les fuerce a reajustar sus sueños (2003: 273). Junto con este aspecto, la tendencia que se da en las parejas jóvenes a sobrevalorar los intereses del varón y a minimizar los de la chica, formaría parte de un modelo sexista de relación que puede acabar derivando en formas explícitas de violencia.

Por otro lado, gran parte de la atención que centran las investigaciones sobre la violencia de género en la adolescencia va dirigida hoy a entender la relación entre la conducta violenta de los chicos y la interiorización de un modelo de masculinidad construido históricamente sobre el rechazo de lo femenino. Algunos estudios realizados con estudiantes de primer y segundo ciclo de Educación Secundaria Obligatoria evidencian un mayor grado de ideología sexista por parte de los chicos, en comparación con el de ellas (Díaz-Aguado, 2003; Lameiras et al., 2009a, 2009b; Meras, 2003).

Los mismos estudios destacan que la construcción de la identidad masculina a partir de elementos sexistas impide que puedan establecerse de forma natural relaciones igualitarias con las chicas. Desde este enfoque, la presión que conlleva la masculinidad se considera muy fuerte y represiva para el menor. En casos extremos, esta presión aumentaría la probabilidad de la expresión de violencia por parte de los chicos, tanto por considerarse una conducta coherente con su rol, como por ser resultado de la acumulación de tensión que implica la represión de sus emociones naturales. Hay que tener en cuenta que entre los rasgos, actitudes y conductas que los chicos aprenden e incorporan a su personalidad en esta etapa destacan la demostración de 
un continuo ejercicio de poder (sobre las mujeres y sobre los hombres que no encajan en el modelo de masculinidad dominante), la ocultación de sus sentimientos y emociones, la indiferencia, las prácticas temerarias y la manifestación de una sexualidad agresiva en la que la figura femenina se convierte en garante de la masculinidad mediante su dominio (Lomas, 2004; Olavarría, 2004; Asturias, 2004).

La escuela se considera además el primer espacio en el que los chicos se verán presionados a adoptar el modelo masculino tradicional, buscando ser recompensados por una cultura en la que los valores ligados a este modelo aún están fuertemente arraigados y son percibidos como "normales" cuando se asignan a la población masculina, aunque desde algunas esferas sociales hayan empezado a cuestionarse. Carlos Lomas lo explica así:

“[...] la cultura masculina del patio y de la escuela constituye un espacio simbólico habitado por una serie de líderes cuyas conductas (con respecto a sus compañeros y a sus compañeras) son un fiel reflejo de las conductas y de los valores asociados al modelo dominante de la masculinidad (el valor absoluto e incuestionable de la fuerza, el elogio de la violencia, el menosprecio del diálogo y de la solidaridad, el maltrato a las chicas y a los chicos que no se identifican con ese modelo dominante de masculinidad...)"(Lomas, 2004: 21-22).

La adopción de una identidad masculina unida al sexismo vendría garantizada por la existencia de una serie de elementos que actuarían como refuerzo de la masculinidad tradicional al proporcionar a los chicos un apoyo social tácito. Así, con el fin de reafirmar su identidad y de conseguir éxito social, el adolescente puede llegar a asimilar la misoginia y la homofobia (rechazo a lo femenino personificado por la figura del varón) (Pescador, 2004: 134) como elementos propios del universo masculino, obteniendo a través de ellos una mayor autoestima y estatus con respecto a las chicas.

Apoyado en este esquema, la reducción de lo femenino a la condición de objeto sexual es asimismo un fenómeno que afecta en la actualidad a la formación de las identidades de género. El informe "La violencia de género en las mujeres jóvenes" ofrece un dato llamativo respecto a la imagen de las mujeres que tienen los chicos en la adolescencia. La descripción que hacen los chicos de las mujeres corresponde claramente a la imagen de objeto sexual difundida por la pornografía (2005: 12). Además, el amor dentro de los parámetros de una masculinidad rígida puede acabar resultando un concepto problemático, por su condición de 
emoción y sentimiento que expresa necesidad y vulnerabilidad, al tiempo que las relaciones recíprocas o igualitarias no serían compatibles con una identidad dependiente del poder y de la demostración de superioridad.

La idealización de la mujer a partir de rasgos que interpretan lo femenino subordinado y devaluado como elemento básico para el desarrollo de la identidad masculina, contrasta con la idealización positiva que paralelamente las chicas tienen de la figura masculina y de la pareja, fenómeno también fomentado por los productos culturales que consumen las adolescentes. El estudio de Ana Meras detecta que entre un $15 \%$ y un $25 \%$ de las chicas tiende a idealizar a su pareja masculina como alguien que les dará felicidad, seguridad y protección, otorgándole una posición superior y reafirmándose ellas mismas como "seres desvalidos" (Meras, 2003: 149).

Meras, haciendo alusión a los cambios acontecidos en el rol femenino, considera que éstos representan una adaptación formal del rol, más que una ruptura que permita hablar de nuevos modelos en las relaciones de género, ya que si bien es cierto que la mujer tiene un acceso incuestionable al trabajo fuera del hogar, sobre ella recaen también las responsabilidades domésticas y de cuidado, aspecto que estructura irremediablemente las relaciones entre hombres y mujeres (2003: 147).

Otra de las cuestiones en las que incide el informe "La violencia de género en las mujeres jóvenes" es la ausencia total de valores en la pareja más allá de los derivados del sistema de género. Los/las adolescentes no vinculan las relaciones afectivo-sexuales con actitudes como el respeto, la tolerancia, la honestidad o la reciprocidad, sino que orientan sus expectativas hacia una figura femenina/masculina, dependiente, como hemos visto, de los estereotipos sexuales válidos dentro del esquema de género aprendido (Comisión para la investigación de malos tratos a mujeres, 2005: 12). Según este informe, la frecuencia con la que se da una idealización excesiva del otro, junto con la ausencia de conocimiento real del amor y de las relaciones de pareja, tendería a producir ideas disfuncionales, especialmente relevantes en el caso de las chicas, al estar vinculadas con la depresión, la dependencia y la baja autoestima.

En resumen, son muchos los análisis que detectan dos sistemas de valores y actitudes claramente diferenciales en función del género, cuya interiorización y reproducción adquiere especial relevancia a partir de la adolescencia. En el modelo femenino habría que valorar la importancia que adquiere una lógica romántica que reserva para las mujeres la experiencia del sacrificio y la dependencia, haciendo que éstas también idealicen la superioridad masculina. En 
el modelo masculino, la socialización de género estaría marcada por la inferiorización de lo femenino, convertido frecuentemente en objeto sexual, y unido simbólicamente a la falta de autoridad y poder. De estos datos se deduce que en la actualidad los/las adolescentes siguen estando expuestos/as a unos roles sexuales rígidos que pueden acabar determinando la forma que adoptarán sus relaciones de pareja.

No obstante, también quisiera hacer hincapié en la creciente heterogeneidad que caracteriza a la realidad española en cuanto a actitudes hacia la igualdad de género, pues, en general, las nuevas formas de establecer una relación de pareja, a partir de valores igualitarios, son percibidas de forma positiva por la sociedad (CIS, 2004a, 2004b). Especialmente en la población femenina se aprecia un mayor rechazo a los valores tradicionales ligados a la desigualdad de género.

El estudio Las mujeres jóvenes en España (2003), realizado por Alberdi, Escario y Matas, aporta información sobre cómo las mujeres jóvenes, a través de sus relaciones personales y motivadas por un cambio profundo de mentalidades con respecto a sus madres, están construyendo unas nuevas relaciones con los hombres en las que domina la expectativa de reciprocidad.

La libertad, la independencia económica y el individualismo aparecen en este estudio como rasgos totalmente asimilados por las mujeres, centrales en sus comportamientos y actitudes hacia sus parejas, si bien las autoras notan la manifestación de algunas contradicciones. Las mujeres se encuentran ante el reto de poder armonizar la igualdad vivida o idealizada en sus relaciones sociales con las diferencias que todavía encuentran en sus relaciones de pareja o que asignan a los valores femeninos y masculinos, algo que marcaría su experiencia emocional.

La investigación de Alberdi, Escario y Matas observa una tendencia en las mujeres jóvenes españolas a la aceptación de dichas contradicciones como fuente de enriquecimiento personal, al tiempo que se abandonan los estereotipos más tradicionales de la identidad femenina. 


\section{Imaginarios sociales del amor de pareja heterosexual en el contexto universitario.}

\section{Metodología}

Los datos que presenta este análisis corresponden a la explotación de 480 cuestionarios, distribuidos en diferentes centros universitarios de la provincia de Alicante $^{1}$, entre los años 2010 y 2012. La edad media de los/las participantes es de 24,82 años, siendo el 59,6\% mujeres y el $40,4 \%$ hombres. Al constituir una población vinculada a la universidad, ésta se caracterizaría por una cierta homogeneidad. Por ejemplo, uno de los rasgos generales de esta muestra tiene que ver con el estado civil, comprobando que el $83,3 \%$ de los/las participantes están solteros/as y que el $87,7 \%$ no tienen hijos/as. En cuanto a la ocupación, el 71,3\% se dedica de forma exclusiva al estudio, el 25,8\% combina éste con un trabajo remunerado, el 2,5\% se encuentra en situación de desempleo y el 0,4\% combina el estudio con labores del hogar.

El objetivo de este cuestionario fue el de acceder a un número suficiente de participantes que luego serían entrevistados/as, y a su vez orientar, aportando información cuantitativa, algunas líneas de una investigación desarrollada como tesis doctoral sobre el amor y las relaciones de pareja desde la perspectiva de género. Este aspecto hacía que el interés principal de estos resultados se circunscribiera a unos determinados elementos del análisis sobre los que resultaba interesante observar si existen diferencias de género, aunque la obtención de datos relevantes en relación con las cuestiones investigadas dio lugar a algunas reflexiones. Estos elementos se recogen en cuatro apartados:

1) Imaginario social del amor

2) Actitud con respecto a aspectos inherentes a la relación de pareja

3) Experiencias positivas y negativas en el contexto de una relación de pareja

4) Valoración de algunos mitos y creencias con respecto a las relaciones de pareja

El cuestionario se diseñó a partir de la operativización de una serie de variables de acuerdo con los objetivos perseguidos por la investigación, en un proceso de consulta recurrente a documentación científica especializada y a diferentes informes correspondientes a otras investigaciones realizadas sobre el tema de la pareja. Para la explotación de datos se utilizó el

1 Participaron concretamente los Departamentos de Ciencias Sociales y Humanas, Tecnología Agroalimentaria y Ciencia Jurídica de la Universidad Miguel Hernández de Elche, los Departamentos de Sociología, Antropología y Economía de la Universidad de Alicante, y la sede de Elda de la UNED (curso de acceso a mayores de 25 años). 
paquete de análisis estadístico SPSS 15.0. Al tratarse de un muestreo intencional estratificado por sexo se optó por trabajar con un nivel de confianza del 0,9544, que implica que con un tamaño muestral de 480 encuestas la probabilidad de acertar en las estimaciones (coincidencia entre el comportamiento de los parámetros analizados en la población real total en relación con la muestra de personas que contesta a la encuesta) se producirá en el 95,44\% de las ocasiones, y que el riesgo estadístico de error se reduce al 4,56\% de los casos (Rodríguez, 1993: 51-55).

\section{Análisis de resultados}

\subsection{Imaginario social del amor}

Uno de los objetivos específicos de este cuestionario fue obtener datos cuantitativos sobre el imaginario colectivo de la pareja heterosexual, y sobre su relación con el género, por eso en las primeras preguntas se solicitó a las/los participantes que escribieran dos rasgos deseables y dos no deseables del hombre o mujer ideal ${ }^{2}$, así como de una relación de pareja ideal. Las respuestas que se obtuvieron en este apartado fueron heterogéneas y no permiten constatar una asociación exclusiva de la feminidad y la masculinidad con los rasgos que tradicionalmente han sido asignados a los sexos, notando además que la mayoría de los calificativos empleados aparecieron con una distribución similar en ambos grupos.

No obstante, sería posible hacer algunas matizaciones. Respecto a los rasgos deseables en hombres y mujeres aparecidos con mayor frecuencia en las respuestas se puede destacar la presencia más contundente de cualidades físicas en la descripción de la mujer ideal hecha por los hombres, en comparación con las cualidades ideales que ellas les asignarían a ellos (tabla 1$)^{3}$. En las respuestas dadas por los hombres guapa representa un $12,2 \%$ sobre el total y deseable un $2,4 \%$, mientras que los calificativos guapo y alto, anotados por las mujeres, constituyen respectivamente un $3,8 \%$ y un $1,8 \%$ del conjunto sus respuestas. La cualidad de atractivo aparece en un porcentaje mayor en las respuestas femeninas, si bien su carácter más subjetivo hace que en esta categoría se puedan incluir rasgos que no han de ser exclusivamente físicos.

\footnotetext{
${ }^{2}$ Se ha de notar que el estudio en el que se enmarca este análisis se centra exclusivamente en parejas heterosexuales, por lo que este requisito impuso un filtro que se aplicó posteriormente a la recogida de los cuestionarios.

${ }^{3}$ Las tablas en este apartado sólo recogen las respuestas aparecidas en mayores porcentajes.
} 
Se puede notar, al mismo tiempo, que aparecen otros muchos rasgos comunes en ambos grupos de respuestas. Mujeres y hombres esperan que sus parejas sean sinceras, observando que esta cualidad ocupa un $11,7 \%$ del total de las apuntadas en las respuestas femeninas y un 9,2\% de las masculinas, siendo también significativa la presencia de la inteligencia entre estas cualidades, que llegaría a representar un $13,6 \%$ de las mencionadas por los hombres. La similitud se hace igualmente patente en los rasgos no deseables asignados a hombres y mujeres. En este caso, los celos, las mentiras y el egoísmo aparecen como los defectos más rechazados tanto por las mujeres como por los hombres, aunque la mayor importancia dada por los varones al aspecto físico vuelve a quedar reflejada en algunos calificativos (fea, gorda) que, sin embargo, no son representativos en las respuestas femeninas (tabla 2). En particular, en el caso de las mujeres se valora negativamente que el hombre sea agresivo $(3,7 \%)$, machista $(3,3 \%)$ y violento $(3,1 \%)$, lo que hace posible apreciar un cierto rechazo de los estereotipos negativos asignados a la masculinidad desde esquemas tradicionales. En las respuestas de los hombres, el rechazo a la pasividad $(2,3 \%)$ de las mujeres, junto a la valoración positiva de la inteligencia como rasgo ideal, también podría estar reflejando esta tendencia a la superación de los roles tradicionales.

Tabla 1. Rasgos deseables en hombre/mujer ideal aparecidos con mayor frecuencia

\begin{tabular}{|c|c|c|c|}
\hline $\begin{array}{c}\text { Rasgos deseables } \\
\text { en hombres }\end{array}$ & $\begin{array}{c}\text { Porcentaje de } \\
\text { respuestas } \\
\text { (mujeres) }\end{array}$ & $\begin{array}{c}\text { Rasgos deseables en } \\
\text { mujeres }\end{array}$ & $\begin{array}{c}\text { Porcentaje de } \\
\text { respuestas } \\
\text { (hombres) }\end{array}$ \\
\hline sincero & 11,7 & inteligente & 13,6 \\
cariñoso & 9,2 & guapa & 12,2 \\
sentido del humor & 6,1 & simpática & 9,5 \\
inteligente & 5,9 & sincera & 9,2 \\
comprensivo & 5,6 & cariñosa & 6,0 \\
guapo & 3,8 & comprensiva & 3,0 \\
respetuoso & 3,8 & divertida & 3,0 \\
fiel & 3,6 & alegre & 2,7 \\
simpático & 3,4 & sentido del humor & 2,7 \\
atractivo & 3,2 & deseable & 2,4 \\
bueno & 2,5 & fiel & 1,9 \\
atento & 2,2 & amable & 1,9 \\
alegre & 2,2 & optimista & 1,9 \\
alto & 1,8 & atractiva & 1,6 \\
educado & 1,8 & buena & 1,6 \\
responsable & 1,8 & respetuosa & 1,1 \\
amable & 1,6 & leal & 1,1 \\
\hline
\end{tabular}

Fuente: Elaboración propia 
Tabla 2. Rasgos no deseables en hombre/mujer ideal aparecidos con mayor frecuencia

\begin{tabular}{|c|c|c|c|}
\hline $\begin{array}{c}\text { Rasgos no } \\
\text { deseables en } \\
\text { hombres }\end{array}$ & $\begin{array}{c}\text { Porcentaje de } \\
\text { respuestas } \\
\text { (mujeres) }\end{array}$ & $\begin{array}{c}\text { Rasgos no } \\
\text { deseables en } \\
\text { mujeres }\end{array}$ & $\begin{array}{c}\text { Porcentaje de } \\
\text { respuestas } \\
\text { (hombres) }\end{array}$ \\
\hline celoso & 6,3 & celosa & 9,7 \\
mentiroso & 5,3 & mentirosa & 5,7 \\
egoísta & 5,3 & egoísta & 4,9 \\
falso & 5,3 & antipática & 3,7 \\
prepotente & 5,1 & fea & 3,4 \\
infiel & 3,9 & infiel & 3,4 \\
agresivo & 3,7 & estúpida & 2,9 \\
machista & 3,3 & prepotente & 2,9 \\
violento & 3,1 & conflictiva & 2,6 \\
conflictivo & 2,9 & superficial & 2,6 \\
posesivo & 2,6 & pasiva & 2,3 \\
frío & 1,5 & mala & 2,3 \\
sucio & 1,5 & gorda & 2,3 \\
sin educación & 1,5 & falsa & 2,3 \\
antipático & 1,3 & creída & 2,0 \\
\hline
\end{tabular}

Fuente: Elaboración propia

En cuanto a los rasgos deseables y no deseables en una relación de pareja ideal, se ha detectado asimismo una relativa igualdad en las respuestas femeninas y masculinas, que trasladan una valoración positiva general de la confianza, el respeto, la sinceridad, el amor y el cariño. La libertad también tendría un porcentaje significativo de respuestas en ambos grupos, aunque la igualdad específicamente sería una característica con presencia en las respuestas femeninas pero no en las masculinas (tabla 3).

Entre los rasgos más señalados por las mujeres, la estabilidad aparece en tercer lugar, siendo el doble de representativa que en el caso de los hombres, aunque en las respuestas masculinas el compromiso y el matrimonio tienen una mayor presencia, lo que hace que los rasgos asociados al ideal de relación de pareja no difieran de forma significativa. 
Tabla 3. Rasgos deseables en la relación de pareja ideal aparecidos con mayor frecuencia

\begin{tabular}{|c|c|c|c|}
\hline Rasgos deseables & $\begin{array}{c}\text { Porcentaje de } \\
\text { respuestas } \\
\text { (mujeres) }\end{array}$ & $\begin{array}{c}\text { Rasgos } \\
\text { deseables }\end{array}$ & $\begin{array}{c}\text { Porcentaje de } \\
\text { respuestas } \\
\text { (hombres) }\end{array}$ \\
\hline confianza & 13,7 & confianza & 12,5 \\
respeto & 8,9 & respeto & 8,4 \\
estabilidad & 7,2 & sinceridad & 7,8 \\
sinceridad & 6,5 & amor & 5,5 \\
comprensión & 5,9 & cariño & 4,4 \\
amor & 5,1 & estabilidad & 3,8 \\
cariño & 4,6 & complicidad & 3,2 \\
complicidad & 3,8 & comunicación & 3,2 \\
comunicación & 3,8 & compromiso & 2,3 \\
fidelidad & 3,2 & diversión & 2,0 \\
amistad & 3,1 & generosidad & 2,0 \\
libertad & 2,5 & libertad & 2,0 \\
pasión & 1,9 & pasión & 2,0 \\
igualdad & 1,7 & sexo & 1,7 \\
compromiso & 1,5 & matrimonio & 1,7 \\
generosidad & 1,3 & fidelidad & 1,7 \\
\hline
\end{tabular}

Fuente: Elaboración propia

Por otro lado, la valoración negativa del no compromiso, de la independencia dentro de la relación de pareja y de la distancia aparece de forma exclusiva en las respuestas masculinas (con los porcentajes $1,2 \%, 1,5 \%$ y $1,2 \%$ respectivamente, tabla 4 ), mientras que el control o el maltrato formarían parte exclusivamente de las respuestas femeninas (con los porcentajes 2,1\% y 1,4\%, tabla 4). El resto de rasgos señalados como no deseables en una relación de pareja se distribuyen de forma similar en las respuestas femeninas y masculinas, con excepción de las mentiras, rasgo que rechazan un $10,3 \%$ de los hombres frente a un 3,7\% de las mujeres. Entre los rasgos con presencia más significativa en ambos grupos con relación a lo no deseable en una relación, la infidelidad, la monotonía y la desconfianza serían los más representativos, lo que trasladaría una idea de la relación a partir de la complicidad y el deseo de felicidad y comunicación.

No obstante, hay que señalar que la igualdad aparecida en este apartado hace referencia especialmente al modo en que mujeres y hombres valoran lo positivo y lo negativo en una pareja, algo que afecta al ámbito de sus expectativas, con independencia de otras posibles valoraciones que puedan hacerse sobre el modo en que ellos/as mismos/as han interiorizado el género. Este aspecto, junto con la edad, puede explicar la menor idealización de rasgos vinculados al modelo tradicional en comparación con los estudios realizados con población adolescente. 
Tabla 4. Rasgos no deseables en la relación de pareja ideal aparecidos con mayor frecuencia

\begin{tabular}{|c|c|c|c|}
\hline Rasgos no deseables & $\begin{array}{c}\text { Porcentaje de } \\
\text { respuestas (mujeres) }\end{array}$ & $\begin{array}{c}\text { Porcentaje de } \\
\text { Rasgos no deseables }\end{array}$ & respuestas (hombres) \\
\hline infidelidad & 7,6 & mentiras & 10,3 \\
celos & 7,6 & infidelidad & 7,0 \\
falsedad & 5,4 & desconfianza & 6,1 \\
monotonía & 5,3 & conflictividad & 5,5 \\
desconfianza & 5,2 & celos & 5,2 \\
mentiras & 3,7 & monotonía & 4,5 \\
no respeto & 3,9 & no comunicación & 3,9 \\
dependencia & 3,3 & falsedad & 3,0 \\
conflictividad & 3,1 & egoísmo & 2,7 \\
no comunicación & 2,9 & posesividad & 2,7 \\
egoísmo & 2,7 & indiferencia & 2,4 \\
indiferencia & 2,5 & no respeto & 1,8 \\
incomprensión & 2,5 & agobio & 1,8 \\
control & 2,1 & independencia & 1,5 \\
agobio & 1,9 & dependencia & 1,5 \\
posesividad & 1,4 & sin sexo & 1,5 \\
maltrato & 1,4 & no compromiso & 1,2 \\
interés & 1,4 & distancia & 1,2 \\
\hline
\end{tabular}

Fuente: Elaboración propia

\subsection{Actitud con respecto a aspectos inherentes a la relación de pareja}

En segundo lugar, se propuso una escala de actitud con respecto a algunos aspectos inherentes a la relación de pareja, cuyo interés era principalmente detectar la posible presencia de diferencias de género. En esta parte del cuestionario se preguntaba por el grado de identificación con algunas afirmaciones mediante las opciones "Muy de acuerdo", "De acuerdo", "En término medio", "En desacuerdo", "Muy en desacuerdo" y "No sabe/ No contesta". Estas afirmaciones hacen referencia a cuestiones que a menudo aparecen atravesadas por el género, siendo interpretadas como femeninas o masculinas. Entre los aspectos que culturalmente se encuentran unidos a lo femenino se han incluido la necesidad de complacer, la vulnerabilidad, la belleza, el miedo a la soledad, el sacrificio o la comunicación. Como actitudes estereotípicas de lo masculino se incluyen el miedo a la intimidad, la fortaleza o la valoración de la belleza física. En contra de lo esperado, en la mayoría de los ítems se han obtenido resultados muy heterogéneos que reflejarían una débil influencia del sistema de género, aunque también es cierto que las diferencias halladas serían coherentes con los significados dominantes de la feminidad/masculinidad en nuestra cultura. Por ejemplo, se aprecian diferencias leves en 
el más alto grado de vulnerabilidad expresado por las mujeres, o la mayor tendencia de éstas a hablar de sentimientos (aunque simultáneamente el 57,3\% de las mujeres y el $53,6 \%$ de los hombres se consideran personas fuertes e independientes). En la tabla 5 se puede observar que el 16,4\% de las mujeres estaría "de acuerdo" o "muy de acuerdo" con el ítem "me siento la parte más vulnerable de la pareja" frente al 10,8\% de los hombres, y un $75,9 \%$ de ellas lo haría con el ítem "hablo con mi pareja de mis sentimientos para solucionar problemas", frente al $63,9 \%$ de los hombres que se identifican en el mismo grado con esta cuestión.

Tabla 5. Identificación Ítems * Sexo

\begin{tabular}{|c|c|c|c|}
\hline "me siento la parte más vulnerable de la pareja" & $\%$ de Mujeres & $\%$ de Hombres & $\%$ del total \\
\hline $\begin{array}{c}\text { Muy en desacuerdo } \\
\text { En desacuerdo } \\
\text { Término medio } \\
\text { De acuerdo } \\
\text { Muy de acuerdo } \\
\text { No sabe/ NC } \\
\text { Total } \\
\end{array}$ & $\begin{array}{c}31,8 \\
17,8 \\
26,6 \\
10,8 \\
5,6 \\
7,3 \\
100,0 \\
\end{array}$ & $\begin{array}{c}29,9 \\
29,4 \\
20,1 \\
7,7 \\
3,1 \\
9,8 \\
100,0 \\
\end{array}$ & $\begin{array}{c}31,0 \\
22,5 \\
24,0 \\
9,6 \\
4,6 \\
8,3 \\
100,0 \\
\end{array}$ \\
\hline $\begin{array}{c}\text { "hablo con mi pareja de mis sentimientos para } \\
\text { solucionar problemas" }\end{array}$ & $\%$ de Mujeres & $\%$ de Hombres & $\%$ del total \\
\hline $\begin{array}{l}\text { Muy en desacuerdo } \\
\text { En desacuerdo } \\
\text { Término medio } \\
\text { De acuerdo } \\
\text { Muy de acuerdo } \\
\text { No sabe/ NC } \\
\text { Total } \\
\end{array}$ & $\begin{array}{c}2,8 \\
1,0 \\
17,5 \\
34,3 \\
41,6 \\
2,8 \\
100,0 \\
\end{array}$ & $\begin{array}{c}3,1 \\
8,2 \\
21,6 \\
38,1 \\
25,8 \\
3,1 \\
100,0 \\
\end{array}$ & $\begin{array}{c}2,9 \\
4,0 \\
19,2 \\
35,8 \\
35,2 \\
2,9 \\
100,0 \\
\end{array}$ \\
\hline $\begin{array}{l}\text { "a veces digo cosas que no pienso para que mi } \\
\text { pareja me respete" }\end{array}$ & $\%$ de Mujeres & $\%$ de Hombres & $\%$ del total \\
\hline $\begin{array}{l}\text { Muy en desacuerdo } \\
\text { En desacuerdo } \\
\text { Término medio } \\
\text { De acuerdo } \\
\text { Muy de acuerdo } \\
\text { No sabe/ NC } \\
\text { Total } \\
\end{array}$ & $\begin{array}{c}56,3 \\
27,3 \\
9,1 \\
4,2 \\
0,3 \\
2,8 \\
100,0 \\
\end{array}$ & $\begin{array}{c}39,7 \\
37,1 \\
11,9 \\
6,7 \\
0,5 \\
4,1 \\
100,0 \\
\end{array}$ & $\begin{array}{c}49,6 \\
31,3 \\
10,2 \\
5,2 \\
0,4 \\
3,3 \\
100,0 \\
\end{array}$ \\
\hline "necesito tener pareja para no sentirme solo/a" & $\%$ de Mujeres & $\%$ de Hombres & $\%$ del total \\
\hline $\begin{array}{c}\text { Muy en desacuerdo } \\
\text { En desacuerdo } \\
\text { Término medio } \\
\text { De acuerdo } \\
\text { Muy de acuerdo } \\
\text { No sabe/ NC } \\
\text { Total } \\
\end{array}$ & $\begin{array}{c}55,2 \\
19,2 \\
14,7 \\
5,9 \\
1,4 \\
3,5 \\
100,0 \\
\end{array}$ & $\begin{array}{c}36,1 \\
33,5 \\
16,5 \\
6,7 \\
2,6 \\
4,6 \\
100,0\end{array}$ & $\begin{array}{c}47,5 \\
25,0 \\
15,4 \\
6,3 \\
1,9 \\
4,0 \\
100,0 \\
\end{array}$ \\
\hline
\end{tabular}

Fuente: Elaboración propia 
Estudios realizados exclusivamente con adolescentes obtienen resultados similares en lo relacionado con la expresión emocional, aunque con diferencias más acentuadas entre chicos y chicas, sobre todo en el mayor grado de sufrimiento e inseguridad de ellas (Caro, 2007: 63-74) y en la más destacada tendencia también de las chicas al sacrificio frente a la mayor contención emocional y expresión de celos por parte de los chicos (Moreno, González y Ros, 2007: 21-34). En los resultados de este cuestionario, aunque sí se ha reflejado una mayor identificación de las mujeres al hacer referencia a la necesidad de comunicación (tabla 5), no se ha podido establecer una asociación entre otros aspectos estudiados, tales como el sacrificio, la culpa o la necesidad de complacer, con una pauta de género. Sí ha sido posible detectar una diferencia de género significativa en el ítem "a veces digo cosas que no pienso para que mi pareja me respete", notando que la opción "Muy en desacuerdo" fue elegida por el 56,3\% de las mujeres y el 39,7\% de los hombres (tabla 5). De la misma forma, tampoco aparece una mayor tendencia femenina al establecimiento de relaciones de pareja con el objetivo de evitar la soledad. Sólo un 7,3\% de las mujeres declara necesitar pareja para no sentirse sola frente a un 10,3\% de los hombres (tabla 5).

Tabla 6. Identificación Ítems * Sexo

\begin{tabular}{|c|c|c|c|}
\hline "elijo a mi pareja por su belleza física" & \% de Mujeres & \% de Hombres & $\%$ del total \\
\hline Muy en desacuerdo & 24,8 & 6,2 & 17,3 \\
En desacuerdo & 20,6 & 5,7 & 14,6 \\
Término medio & 38,5 & 34,5 & 36,9 \\
De acuerdo & 10,8 & 41,2 & 23,1 \\
Muy de acuerdo & 3,1 & 9,8 & 5,8 \\
No sabe/ NC & 2,1 & 2,6 & 2,3 \\
Total & 100,0 & 100,0 & 100,0 \\
\hline "elijo a mi pareja por la admiración que siento & & & \\
por ella" & $\%$ de Mujeres & $\%$ de Hombres & $\%$ del total \\
\hline Muy en desacuerdo & 7,3 & 5,2 & 6,5 \\
En desacuerdo & 10,1 & 7,7 & 9,2 \\
Término medio & 30,4 & 23,7 & 27,7 \\
De acuerdo & 27,3 & 41,2 & 32,9 \\
Muy de acuerdo & 18,5 & 19,1 & 18,8 \\
No sabe/ NC & 6,3 & 3,1 & 5,0 \\
Total & 100,0 & 100,0 & 100,0 \\
\hline "me enfado cuando mi pareja no responde & & & \\
como yo esperaba" & $\%$ de Mujeres & $\%$ de Hombres & $\%$ del total \\
\hline Muy en desacuerdo & 9,4 & 14,9 & 11,7 \\
En desacuerdo & 16,4 & 21,1 & 18,3 \\
Término medio & 39,2 & 45,4 & 41,7 \\
De acuerdo & 20,3 & 11,9 & 16,9 \\
Muy de acuerdo & 11,2 & 3,6 & 8,1 \\
No sabe/ NC & 3,5 & 3,1 & 3,3 \\
Total & 100,0 & 100,0 & 100,0 \\
\hline
\end{tabular}

Fuente: Elaboración propia 
Por otro lado, las respuestas femeninas reflejan un menor nivel de exigencia en cuanto a la belleza física o a la admiración como condición determinante en la elección de pareja, a la vez que unas mayores expectativas con respecto a la relación. Éstas son las actitudes en las que se aprecia una mayor diferencia en las puntuaciones de mujeres y hombres, siendo especialmente destacable en el ítem "elijo a mi pareja por su belleza física", en el que la mitad de los hombres (51\%) responde estar "de acuerdo" o "muy de acuerdo" frente al 13,9\% de las mujeres (tabla 6). En el ítem "elijo a mi pareja por la admiración que siento por ella" las respuestas femeninas afirmativas siguen siendo más bajas (45,8\% frente al 60,3\% de las respuestas masculinas, tabla 6). Las mayores expectativas de las mujeres con respecto a la relación de pareja quedan reflejadas en el mayor porcentaje de éstas en las respuestas "de acuerdo" o "muy de acuerdo" en el ítem "me enfado cuando mi pareja no responde como yo esperaba" (31,5\% frente al 15,5\% de las respuestas masculinas, tabla 6).

En este apartado se han introducido a su vez algunos aspectos inherentes a las relaciones humanas que pueden estar presentes en las relaciones de pareja, como la libertad o la confianza, recogidos en los ítems "me gusta dejar espacio a mi pareja para que no se agobie" y "mi pareja es la persona en la que más confío" (tabla 7). En estos casos los porcentajes de respuestas afirmativas totales son de $71,5 \%$ y $70,3 \%$ respectivamente, lo que reafirma un imaginario del amor influido por igual por el individualismo y el deseo de intimidad, sin hallarse diferencias de género.

Tabla 7. Identificación Ítems $*$ Sexo

\begin{tabular}{|c|c|c|c|}
\hline "me gusta dejar espacio a mi & & & \\
pareja para que no se agobie" & \% de Mujeres & \% de Hombres & \% del total \\
\hline Muy en desacuerdo & 0,7 & 1,0 & 0,8 \\
En desacuerdo & 3,5 & 4,1 & 3,8 \\
Término medio & 20,6 & 20,6 & 20,6 \\
De acuerdo & 33,9 & 35,1 & 34,4 \\
Muy de acuerdo & 38,5 & 35,1 & 37,1 \\
No sabe/ NC & 2,8 & 4,1 & 3,3 \\
Total & 100,0 & 100,0 & 100,0 \\
\hline "mi pareja es la persona en la & & & \\
que más confío" & $\%$ de Mujeres & $\%$ de Hombres & $\%$ del total \\
\hline Muy en desacuerdo & 3,5 & 4,1 & 3,8 \\
En desacuerdo & 3,8 & 5,7 & 4,6 \\
Término medio & 18,5 & 14,9 & 17,1 \\
De acuerdo & 28,7 & 28,9 & 28,8 \\
Muy de acuerdo & 40,6 & 42,8 & 41,5 \\
No sabe/ NC & 4,9 & 3,6 & 4,4 \\
Total & 100,0 & 100,0 & 100,0 \\
\hline
\end{tabular}

Fuente: Elaboración propia 


\subsection{Experiencias positivas y negativas en el contexto de una relación de pareja}

Para valorar la interferencia del género en la experiencia de la relación de pareja se propuso a las/los participantes una serie de afirmaciones que hacían referencia a hechos positivos y negativos que pueden tener lugar en el contexto de una relación íntima, teniendo como respuestas las opciones "Nunca", "Una vez", "Varias veces", "Frecuentemente" y "No sabe/ No contesta".

El registro de experiencias positivas y negativas muestra igualmente respuestas similares en ambos grupos que no permiten hacer generalizaciones a partir de estos resultados. En general, la mayoría de personas han experimentado en sus relaciones de pareja aspectos positivos como el cariño, la comprensión, la comunicación, el apoyo, el respeto y una sexualidad satisfactoria.

Todos estos aspectos aparecen en las respuestas obtenidas con frecuencias muy similares en las respuestas femeninas y masculinas. Por ejemplo, el 59,1\% de las mujeres y el $57,7 \%$ de los hombres declaran sentirse escuchadas/os y comprendidas/os frecuentemente en sus relaciones de pareja. Tan sólo un 1,4\% de mujeres y un 1,5\% de hombres responden no haber recibido nunca apoyo en momentos difíciles, y es un porcentaje similar el de mujeres y hombres que tampoco habrían experimentado confianza y seguridad $(2,8 \%$ y $3,6 \%$ respectivamente). Asimismo, disfrutar de la sexualidad con frecuencia en el contexto de la pareja es un aspecto destacado por un $71,7 \%$ de las mujeres y un $69,1 \%$ los hombres.

Las diferencias presentes en las frecuencias de las diferentes opciones propuestas podrían ser, de hecho, más significativas que las halladas por sexo. Es decir, mientras que el $75,4 \%$ de la muestra afirma haber recibido atenciones y cariño por parte de su pareja frecuentemente, sólo el 54,6\% lo hace cuando el ítem propuesto se refiere a la libertad y espacio personal (tabla 8), lo que revela un patrón común en las relaciones de pareja en el que el respeto a la individualidad y a la libertad del otro, a pesar de ser aspectos valorados muy positivamente, tendrían en la práctica una presencia menos importante que el cariño. 
Tabla 8. Identificación Ítems $*$ Sexo

\begin{tabular}{|c|c|c|c|}
\hline $\begin{array}{c}\text { "me ha expresado } \\
\text { atenciones y cariño" }\end{array}$ & \% de Mujeres & \% de Hombres & \% del total \\
\hline Nunca & 0,3 & 0,5 & 0,4 \\
Una vez & 1,0 & 2,1 & 1,5 \\
Varias veces & 21,0 & 20,6 & 20,8 \\
Frecuentemente & 76,6 & 73,7 & 75,4 \\
No sabe/ NC & 1,0 & 3,1 & 1,9 \\
Total & 100,0 & 100,0 & 100,0 \\
\hline "ha respetado mi libertad & & & \\
y espacio personal" & $\%$ de Mujeres & $\%$ de Hombres & $\%$ del total \\
\hline Nunca & 3,1 & 3,6 & 3,3 \\
Una vez & 5,9 & 11,3 & 8,1 \\
Varias veces & 31,5 & 31,4 & 31,5 \\
Frecuentemente & 56,3 & 52,1 & 54,6 \\
No sabe/ NC & 3,1 & 1,5 & 2,5 \\
Total & 100,0 & 100,0 & 100,0 \\
\hline
\end{tabular}

Fuente: Elaboración propia

Por otro lado, las experiencias negativas que se proponían en este cuestionario expresan menores niveles de frecuencia tanto en las respuestas femeninas como en las masculinas, sin encontrarse diferencias de género.

Estas experiencias se recogían en los siguientes ítems: "ha criticado sin motivo mi forma de ser", "me ha obligado a hacer algo", "ha dejado de hablarme o escucharme", "me ha acusado injustamente de algo", "me ha ridiculizado", "ha desvalorizado mis preocupaciones", "se ha enfadado por manifestar mis opiniones", "me ha mentido en algo importante" y "me ha agredido".

Como aspectos afectados por diferencias que pueden conectarse con los estereotipos de género, se puede destacar una mayor percepción de la obligación en la relación de pareja por parte de los varones frente a una percepción algo más acusada de la infravaloración de las propias preocupaciones por parte de las mujeres, sin que las puntuaciones sean especialmente relevantes (tabla 9). 
Tabla 9. Identificación Ítems * Sexo

\begin{tabular}{|c|c|c|c|}
\hline $\begin{array}{c}\text { "me ha obligado a hacer } \\
\text { algo" }\end{array}$ & \% de Mujeres & \% de Hombres & \% del total \\
\hline Nunca & 74,5 & 51,5 & 65,2 \\
Una vez & 9,4 & 12,4 & 10,6 \\
Varias veces & 11,5 & 25,3 & 17,1 \\
Frecuentemente & 1,7 & 3,6 & 2,5 \\
No sabe/ NC & 2,8 & 7,2 & 4,6 \\
Total & 100,0 & 100,0 & 100,0 \\
\hline "ha desvalorizado mis & & & \\
preocupaciones" & $\%$ de Mujeres & $\%$ de Hombres & $\%$ del total \\
\hline Nunca & 52,8 & 59,3 & 55,4 \\
Una vez & 22,4 & 14,4 & 19,2 \\
Varias veces & 16,8 & 17,0 & 16,9 \\
Frecuentemente & 5,6 & 1,5 & 4,0 \\
No sabe/ NC & 2,4 & 7,7 & 4,6 \\
Total & 100,0 & 100,0 & 100,0 \\
\hline
\end{tabular}

Fuente: Elaboración propia

En el resto de los ítems no se han apreciado respuestas que apunten a la existencia de una pauta diferencial de género. Además, en estas cuestiones se registra un elevado porcentaje de la respuesta "No sabe/ NC" y de no respuesta, lo que limita la eficacia de estas preguntas en el objetivo propuesto. No obstante, se ha de contar con que, si bien en formulaciones más o menos abiertas, como son las utilizadas en el presente cuestionario, la tendencia es a puntuaciones similares en los hombres y las mujeres, ante afirmaciones más específicas las respuestas podrían cambiar. Asimismo, las características específicas de esta muestra pueden favorecer un mayor nivel de igualdad con respecto a la población más joven o con menos preparación, pues, como detectan muchos estudios, la intervención de ideología sexista en la experiencia del amor es un fenómeno claramente vigente en la adolescencia.

\subsection{Valoración de algunos mitos y creencias con respecto a las relaciones de pareja}

Los resultados sobre la valoración de algunos mitos y creencias con respecto a las relaciones de pareja provienen de las identificaciones de los/las participantes con algunas afirmaciones, a partir de las opciones de respuesta "Muy de acuerdo", "De acuerdo", "En término medio", "En desacuerdo", "Muy en desacuerdo" y "No sabe/ No contesta".

En términos generales, las respuestas femeninas y masculinas han sido similares en muchos aspectos. Por ejemplo, en la tabla 10 se puede observar un mayor porcentaje de respuestas favorables 
(con respecto a las desfavorables) en el ítem "Hombres y mujeres necesitamos cosas diferentes" (el conjunto de la muestra que está "de acuerdo" o "muy de acuerdo" representa el 35,4\% frente al 24,8\% que está "en desacuerdo" o "muy en desacuerdo"), o que vinculan la atracción con la oposición y complementariedad (29,4\% frente al 22,9\% que rechaza este ítem), así como el amor con lo irracional (58,2\% frente al $19 \%$ que rechaza este ítem). No obstante, en los dos primeros casos la respuesta con un porcentaje más alto sería "término medio". Sí destaca el número mayor de mujeres que piensan que el amor es irracional y que no se puede elegir a quien se ama (61,6\% de ellas frente al 53,1\% de ellos), resultado que coincide con los del estudio de Bosch et al. (2007), que también detectaría una mayor tendencia en la población joven femenina a asumir concepciones más idealizadas por las que se aceptarían más fácilmente los mitos en los que el amor se caracteriza por sus cualidades mágicas (mito de la media naranja, predestinación, etc.).

Tabla 10. Identificación Ítems * Sexo

\begin{tabular}{|c|c|c|c|}
\hline "hombres y mujeres & & & \\
necesitamos cosas diferentes" & \% de Mujeres & \% de Hombres & \% del total \\
\hline Muy en desacuerdo & 7,7 & 9,3 & 8,3 \\
En desacuerdo & 18,2 & 13,9 & 16,5 \\
Término medio & 35,7 & 36,6 & 36,0 \\
De acuerdo & 23,1 & 22,7 & 22,9 \\
Muy de acuerdo & 13,3 & 11,3 & 12,5 \\
No sabe/ NC & 2,1 & 6,2 & 3,8 \\
Total & 100,0 & 100,0 & 100,0 \\
\hline "los opuestos se atraen y & & & \\
complementan" & $\%$ de Mujeres & $\%$ de Hombres & $\%$ del total \\
\hline Muy en desacuerdo & 7,0 & 15,5 & 10,4 \\
En desacuerdo & 11,9 & 13,4 & 12,5 \\
Término medio & 43,0 & 37,1 & 40,6 \\
De acuerdo & 17,8 & 14,4 & 16,5 \\
Muy de acuerdo & 13,3 & 12,4 & 12,9 \\
No sabe/ NC & 7,0 & 7,2 & 7,1 \\
Total & 100,0 & 100,0 & 100,0 \\
\hline "el amor es irracional, no se & & & \\
puede elegir a quién se ama" & $\%$ de Mujeres & $\%$ de Hombres & $\%$ del total \\
\hline Muy en desacuerdo & 5,6 & 8,8 & 6,9 \\
En desacuerdo & 14,0 & 9,3 & 12,1 \\
Término medio & 16,8 & 22,7 & 19,2 \\
De acuerdo & 24,5 & 28,9 & 26,3 \\
Muy de acuerdo & 37,1 & 24,2 & 31,9 \\
No sabe/ NC & 2,1 & 6,2 & 3,8 \\
Total & 100,0 & 100,0 & 100,0 \\
\hline
\end{tabular}

Fuente: Elaboración propia 
También se puede señalar, a partir de estos resultados, que es mayor el número de personas que se muestran favorables a la igualdad en la valoración de la crianza de los hijos/as, siendo el porcentaje de mujeres que están "de acuerdo" o "muy de acuerdo" superior al de los hombres $(92,6 \%$ frente al 83,5\% respectivamente, tabla 11). La distribución de respuestas femeninas y masculinas es también similar ante la afirmación "el fin de la pasión suele ser el fin de la relación de pareja", aunque en este caso es superior el porcentaje de hombres que está "de acuerdo" o "muy de acuerdo" (31\%) que el de mujeres (25,5\%). Y, por otro lado, si bien en resultados anteriores habían destacado las mayores aspiraciones femeninas con respecto a la relación de pareja, al hacer alusión concretamente a la importancia de que tu pareja cumpla tus expectativas para que la relación sea buena la tendencia se invierte y son más los hombres $(41,2 \%)$ que las mujeres (36\%) que responden afirmativamente (tabla 11$)$.

Tabla 11. Identificación Ítems * Sexo

\begin{tabular}{|c|c|c|c|}
\hline $\begin{array}{c}\text { "madre y padre deberían } \\
\text { disfrutar por igual de la } \\
\text { crianza de sus hijos/as" }\end{array}$ & \% de Mujeres & \% de Hombres & \% del total \\
\hline Muy en desacuerdo &, 7 & 2,6 & 1,5 \\
En desacuerdo & 2,4 & 3,1 & 2,7 \\
Término medio & 3,5 & 6,2 & 4,6 \\
De acuerdo & 26,9 & 26,8 & 26,9 \\
Muy de acuerdo & 65,7 & 56,7 & 62,1 \\
No sabe/ NC &, 7 & 4,6 & 2,3 \\
Total & 100,0 & 100,0 & 100,0 \\
\hline "el fin de la pasión suele ser el & & & \\
fin de la relación de pareja" & $\%$ de Mujeres & $\%$ de Hombres & $\%$ del total \\
\hline Muy en desacuerdo & 12,9 & 7,2 & 10,6 \\
En desacuerdo & 21,7 & 20,6 & 21,3 \\
Término medio & 36,4 & 33,0 & 35,0 \\
De acuerdo & 15,7 & 22,2 & 18,3 \\
Muy de acuerdo & 9,8 & 8,8 & 9,4 \\
No sabe/ NC & 3,5 & 8,2 & 5,4 \\
Total & 100,0 & 100,0 & 100,0 \\
\hline "en una relación de pareja & & & \\
buena tu pareja ha de cumplir & & & $\%$ del total \\
tus expectativas" & $\%$ de Mujeres & $\%$ de Hombres & 4,2 \\
\hline Muy en desacuerdo & 3,8 & 4,6 & 12,3 \\
En desacuerdo & 13,3 & 10,8 & 42,1 \\
Término medio & 44,1 & 39,2 & 31,5 \\
De acuerdo & 29,4 & 34,5 & 6,7 \\
Muy de acuerdo & 6,6 & 6,7 & 3,3 \\
No sabe/ NC & 2,8 & 4,1 & 100,0 \\
Total & 100,0 & 100,0 &
\end{tabular}

Fuente: Elaboración propia 
Otro aspecto destacable en este análisis sería la mayor contundencia de las mujeres en el rechazo a la afirmación "cuando una mujer es agredida algo habrá hecho para provocarlo" (tabla 12). En este caso, el porcentaje de mujeres que declara estar "en desacuerdo" o "muy en desacuerdo" con el ítem sería de un 95,5\%, en comparación con el 84,5\% de los hombres ${ }^{4}$. La tendencia a una mayor oposición femenina a los tópicos tradicionales se ha dado también en el ítem "las relaciones amor-odio son fruto de la pasión", rechazado por un 49,6\% de las mujeres y un $37,1 \%$ de los hombres (tabla 12). Esta pauta marca asimismo las respuestas recogidas en el ítem "el matrimonio es el paso más importante en una relación de pareja", con el que casi la mitad de las mujeres (48,6\%) se declara "en desacuerdo" y "muy en desacuerdo" frente al $39,2 \%$ de los hombres. Este dato coincide con los resultados obtenidos en el primer apartado, en el que, a pesar de que un mayor porcentaje de mujeres que de hombres valoraba la estabilidad como rasgo deseable en la relación de pareja, el matrimonio tenía una mayor presencia en las idealizaciones masculinas.

Tabla 12. Identificación Ítems * Sexo

\begin{tabular}{|c|c|c|c|}
\hline $\begin{array}{c}\text { "cuando una mujer es } \\
\text { agredida, algo habrá hecho } \\
\text { para provocarlo" }\end{array}$ & \% de Mujeres & \% de Hombres & \% del total \\
\hline Muy en desacuerdo & 90,6 & 73,7 & 83,8 \\
En desacuerdo & 4,9 & 10,8 & 7,3 \\
Término medio & 1,7 & 5,7 & 3,3 \\
De acuerdo &, 7 & 3,1 & 1,7 \\
Muy de acuerdo &, 3 &, 5 &, 4 \\
No sabe/ NC & 1,7 & 6,2 & 3,5 \\
Total & 100,0 & 100,0 & 100,0 \\
\hline "las relaciones amor-odio son & & & \\
fruto de la pasión" & $\%$ de Mujeres & \% de Hombres & $\%$ del total \\
\hline Muy en desacuerdo & 28,3 & 14,9 & 22,9 \\
En desacuerdo & 21,3 & 22,2 & 21,7 \\
Término medio & 25,5 & 27,3 & 26,3 \\
De acuerdo & 12,6 & 15,5 & 13,8 \\
Muy de acuerdo & 4,9 & 7,7 & 6,0 \\
No sabe/ NC & 7,3 & 12,4 & 9,4 \\
Total & 100,0 & 100,0 & 100,0 \\
\hline
\end{tabular}

\footnotetext{
${ }^{4}$ Estudios con población adolescente también señalan la diferente percepción de la violencia por parte de chicos y chicas (Bosch et al., 2007; Martínez, Bonilla, Gómez y Bayot, 2008), revelando incluso una mayor tolerancia de los varones con respecto a las actitudes relacionadas con la violencia en el contexto de la pareja.
} 


\begin{tabular}{|c|c|c|c|}
\hline $\begin{array}{c}\text { "el matrimonio es el paso más } \\
\text { importante en una relación de } \\
\text { pareja" }\end{array}$ & \% de Mujeres & \% de Hombres & \% del total \\
\hline Muy en desacuerdo & 26,6 & 19,6 & 23,8 \\
En desacuerdo & 22,0 & 19,6 & 21,0 \\
Término medio & 24,1 & 22,7 & 23,5 \\
De acuerdo & 13,6 & 16,5 & 14,8 \\
Muy de acuerdo & 11,9 & 17,0 & 14,0 \\
No sabe/ NC & 1,7 & 4,6 & 2,9 \\
Total & 100,0 & 100,0 & 100,0 \\
\hline "el amor requiere sacrificios" & $\%$ de Mujeres & $\%$ de Hombres & $\%$ del total \\
\hline Muy en desacuerdo & 4,9 & 3,1 & 4,2 \\
En desacuerdo & 11,2 & 9,8 & 10,6 \\
Término medio & 30,8 & 18,0 & 25,6 \\
De acuerdo & 29,7 & 38,1 & 33,1 \\
Muy de acuerdo & 22,4 & 27,8 & 24,6 \\
No sabe/ NC & 1,0 & 3,1 & 1,9 \\
Total & 100,0 & 100,0 & 100,0 \\
\hline
\end{tabular}

Fuente: Elaboración propia

Finalmente, otra de las diferencias de género apreciadas se halla en las respuestas ante la afirmación "el amor requiere sacrificios" (tabla 12), viendo que es mayor el número de hombres $(65,9 \%)$ que declara estar "de acuerdo" o "muy de acuerdo", que el de mujeres $(52,1 \%)$.

\section{Conclusiones}

Al analizar la forma en que se presenta el imaginario colectivo del amor heterosexual en el contexto universitario ha sido posible detectar una menor presencia de diferencias de género con respecto a las encontradas en estudios centrados en población adolescente, siendo más apreciable la tendencia a la igualdad en aspectos tales como la idealización de la relación de pareja sobre la base de la confianza y la complicidad, o en determinadas actitudes y experiencias que conllevan una clara extensión del individualismo y la libertad al terreno de la intimidad.

Hombres y mujeres plantean conceptos parecidos que trasladan una idea de la pareja como asociación estable, en la que lo deseable y lo no deseable estaría más relacionado con la satisfacción y felicidad personal que con los estereotipos de género. 
Entre los rasgos deseables con una valoración más positiva en ambos grupos destacarían la confianza, el respeto, la sinceridad, el amor y el cariño. Con presencia también significativa ha aparecido la libertad como elemento deseable en una relación de pareja, aunque al mismo tiempo, los resultados han reflejado un patrón común de comportamiento en pareja en el que el respeto a la individualidad y a la libertad del otro sería menos relevante que el cariño.

No obstante, ha llamado la atención la presencia de la igualdad como ideal en la relación de pareja exclusivamente en las respuestas femeninas, resultado que se ha dado paralelamente a la mayor contundencia también de las respuestas femeninas en el rechazo a los tópicos negativos asociados con el modelo tradicional de género, como la agresividad, el machismo o el control.

Otro aspecto en el que sí aparece una influencia de los modelos tradicionales de género ha sido la mayor importancia dada por el grupo de los hombres a la belleza física dentro del ideal femenino y como aspecto determinante en la elección de pareja. Este dato contrasta con una baja valoración de la belleza física por parte de las mujeres, aunque éstas reflejarían mayores expectativas en la pareja. En cualquier caso, los resultados sobre actitudes en el marco de las relaciones de pareja reflejan en general diferencias muy leves en las respuestas femeninas y masculinas, que se refuerzan algo más en aspectos como la expresión emocional o el sentimiento de vulnerabilidad, donde los porcentajes más altos corresponden a las mujeres.

En cuanto a las experiencias y conflictos asociados a las relaciones de pareja, no han destacado diferencias significativas por sexo atendiendo a las respuestas dadas en este cuestionario, aunque sí se han registrado diferencias sutiles que han permitido hacer algunas apreciaciones, como el que pueda existir una percepción más acusada de los varones de la obligación en el contexto de la pareja o un sentimiento de infravaloración más frecuente en las mujeres.

Y, por último, las respuestas sobre creencias relacionadas con el amor y la pareja han reflejado una mayor variabilidad que, sin embargo, esconde algunas diferencias importantes marcadas por la variable sexo. Éstas se expresan en los mayores porcentajes de mujeres que idealizan el amor como aspecto irracional, que son favorables a la igualdad en la crianza de los hijos/as y que rechazan la violencia, y en los mayores 
porcentajes de hombres que vinculan la duración de la pareja a la pasión y el amor al sacrificio.

Al mismo tiempo, se ha de notar que la heterogeneidad ha sido característica del conjunto de respuestas, sobre todo, en los posicionamientos ante las creencias y mitos planteados, lo que pone de relieve la necesidad de abordar el tema del amor y la pareja a partir de técnicas cualitativas, dada la dificultad de tratar aspectos tan complejos y subjetivos de la vida humana mediante respuestas estandarizadas.

Más allá de esta apreciación, los resultados aquí recogidos ponen de relieve que la ideología sexista podría estar más presente en el imaginario de la adolescencia que en el de la población universitaria, y que la posición notablemente crítica hacia los tópicos negativos del modelo tradicional, que en este estudio ha formado parte de las respuestas femeninas, podría relacionarse con un ejercicio de reflexión sobre este tema.

\section{BIBLIOGRAFÍA}

- Alberdi, Inés; Escario, Pilar y Matas, Natalia (2003): Las mujeres jóvenes en España. Barcelona: Fundación La Caixa.

- Altable Vicario, Charo (1991): Penélope o las trampas del amor. Por una coeducación sentimental. Madrid: Mare Nostrum Ediciones Didácticas.

- Asturias, Laura E. (2004): “La construcción de la masculinidad y las relaciones de género". En: Carlos Lomas (comp.): Los chicos también lloran. Identidades masculinas, igualdad entre los sexos y coeducación. Barcelona: Paidós Ibérica, pp. 65-78.

- Bosch Fiol, Esperanza et al. (2007): "Del mito del amor romántico a la violencia contra las mujeres en la pareja", 2004-2007, Instituto de la Mujer, Ministerio de Igualdad, [en línea] Disponible en: $\quad$ http://centreantigona.uab.cat/izquierda/amor\%20romantico\%20Esperanza\%20Bosch.pdf [19/12/2013].

- Caro Blanco, Coral (2007): “La concepción romántica del amor trasfondo de la violencia de género". En: Victoria A. Ferrer y Esperanza Bosch (comps.): Los feminismos como herramientas de cambio social (II): De la violencia contra las mujeres a la construcción del pensamiento feminista. Palma de Mallorca: UIB, pp. 63-74. 
- Centro de Investigaciones Sociológicas (2004a): "Opiniones y actitudes sobre la familia", Estudio $\mathrm{n}^{\mathrm{o}}$ 2578, [en línea] Disponible en: http://www.cis.es/cis/opencms/Archivos/Marginales/2560_2579/2578/Es2578.pdf [10/3/2010]. . (2004b): “Actitudes y opiniones de las mujeres ante los hijos y la crianza”, Estudio no 2552, [en línea] Disponible en: http://www.cis.es/cis/opencms/Archivos/Marginales/2540_2559/2552/cues2552.pdf [10/3/2010].

- Colás Bravo, Pilar y Villaciervos Moreno, Patricia (2007): “La interiorización de los estereotipos de género en jóvenes y adolescentes". En: Revista de Investigación Educativa, vol. 25, $\mathrm{n}^{\mathrm{o}} \quad 1, \quad \mathrm{pp}$. 35-58., [en línea] Disponible en: http://revistas.um.es/rie/article/viewFile/96421/92631 [11/3/2010].

- Comisión para la investigación de malos tratos a mujeres (2005): "La violencia de género en las mujeres jóvenes", Madrid, [en línea] Disponible en: http://www.madrid.org/cs/Satellite?blobcol=urldata\&blobheader=application/pdf\&blobkey=id $\underline{\text { \&blobtable}=\text { MungoBlobs \&blobwhere }=1220373748652 \& \text { ssbinary }=\text { true }}[$ [18/11/2013].

- Díaz-Aguado, Ma José (2003): “Adolescencia, sexismo y violencia de género”. En: Papeles del psicólogo, 2003, nº 84, pp. 35-44.

- Díaz Perera, Enriqueta (2009): "Roles y estereotipos que rigen las relaciones entre adolescentes de Bachillerato del área metropolitana de Barcelona". En: VV.AA.: Poder, poderes y empoderamiento... ¿Y el amor? ;Ah, el amor!, Actas $5^{\circ}$ Congreso Estatal Isonomia sobre Igualdad entre mujeres y hombres, Fundación Isonomia, Universitat Jaume I, pp. 215-220, [en línea] Disponible en: http://isonomiafundacion.uji.es/wpcontent/uploads/publicaciones/Actas_Congresos_Estatales/V_Actas.pdf [16/03/2012].

- Giddens, Anthony (1995): La transformación de la intimidad: sexualidad, amor y erotismo en las sociedades modernas. Madrid: Cátedra.

- Lameiras, María, Carrera, Ma Victoria, Rodríguez, Yolanda y Failde, José María (2009a): "Influencia de los mass media en las actitudes sexistas de adolescentes de $2^{\circ}$ Ciclo de Educación Secundaria Obligatoria”. En: VV.AA.: Poder, poderes y empoderamiento... ¿Y el amor? iAh, el amor!, Actas $5^{\circ}$ Congreso Estatal Isonomia sobre Igualdad entre mujeres y hombres, Fundación Isonomia, Universitat Jaume I, pp. 215-220, [en línea] Disponible en: http://isonomiafundacion.uji.es/wpcontent/uploads/publicaciones/Actas_Congresos_Estatales/V_Actas.pdf [16/03/2012]. . (2009b): "Relación entre actitudes sexistas y actitudes hacia la sexualidad en adolescentes de 1er. Ciclo de Educación Secundaria Obligatoria”. En: VV.AA.: Poder, poderes y 
empoderamiento... ¿Y el amor? ;Ah, el amor!, Actas $5^{\circ}$ Congreso Estatal Isonomia sobre Igualdad entre mujeres y hombres, Fundación Isonomia, Universitat Jaume I, pp. 215-220, [en línea] Disponible en: http://isonomiafundacion.uji.es/wpcontent/uploads/publicaciones/Actas_Congresos_Estatales/V_Actas.pdf [16/03/2012].

- Leal García, Aurora (2007): "Nuevos tiempos, viejas preguntas sobre el amor. Un estudio con adolescentes". En: Posgrado y sociedad, vol. 7, n 2, pp. 50-70, [en línea] Disponible en: www.dialnet.unirioja.es/descarga/articulo/3662521.pdf [19/12/2013].

- Lomas, Carlos (2004): “LLos chicos no lloran?”. En: Carlos Lomas (comp.): Los chicos también lloran. Identidades masculinas, igualdad entre los sexos y coeducación. Barcelona: Paidós Ibérica, pp. 9-29.

- Martínez Benlloch, Isabel, Bonilla Campos, Amparo, Gómez Sánchez, Lucía y Bayot, Agustín (2008): "Identidad de género y afectividad en la adolescencia: asimetrías relacionales y violencia simbólica”. En: Anuario de Psicología, vol. 39, nº 1, pp. 109-118.

- Meras Lliebre, Ana (2003): "Prevención de la violencia de género en adolescentes”. En: Estudio de Juventud $\mathrm{n}^{\mathrm{o}}$ 62/03, pp. 143-150, [en línea] Disponible en: http://www.injuve.es/sites/default/files/art11.pdf [11/8/2010].

- Moreno, Monserrat, González, Alba y Ros, Marc (2007): "Enamoramiento y violencia contra las mujeres”. En: Victoria A. Ferrer y Esperanza Bosch (comps.): Los feminismos como herramientas de cambio social (II): De la violencia contra las mujeres a la construcción del pensamiento feminista. Palma de Mallorca: UIB, pp. 21-34.

- Olavarría, José (2004): “Modelos de masculinidad y desigualdades de género”. En: Carlos Lomas (comp.): Los chicos también lloran. Identidades masculinas, igualdad entre los sexos y coeducación. Barcelona: Paidós Ibérica, pp. 45-63.

- Pescador Albiach, Erick (2004): "Masculinidades y adolescencia". En: Carlos Lomas (comp.): Los chicos también lloran. Identidades masculinas, igualdad entre los sexos y coeducación. Barcelona: Paidós Ibérica, pp. 113-146.

- Rodríguez Osuna, Jacinto (1993): Métodos de muestreo. Casos prácticos, Madrid: CIS.

- Sastre Vilarrasa, Genoveva y Moreno Marimón, Monserrat (2003): Resolución de conflictos y aprendizaje emocional. Una perspectiva de género. Barcelona: Editorial Gedisa. 